\title{
Würde versus Gentechnologie?
}

\author{
Sabine Odparlik · Peter Kunzmann
}

Received: 9 July 2009/Published online: 6 August 2009

(c) Birkhäuser Verlag, Basel/Switzerland 2009

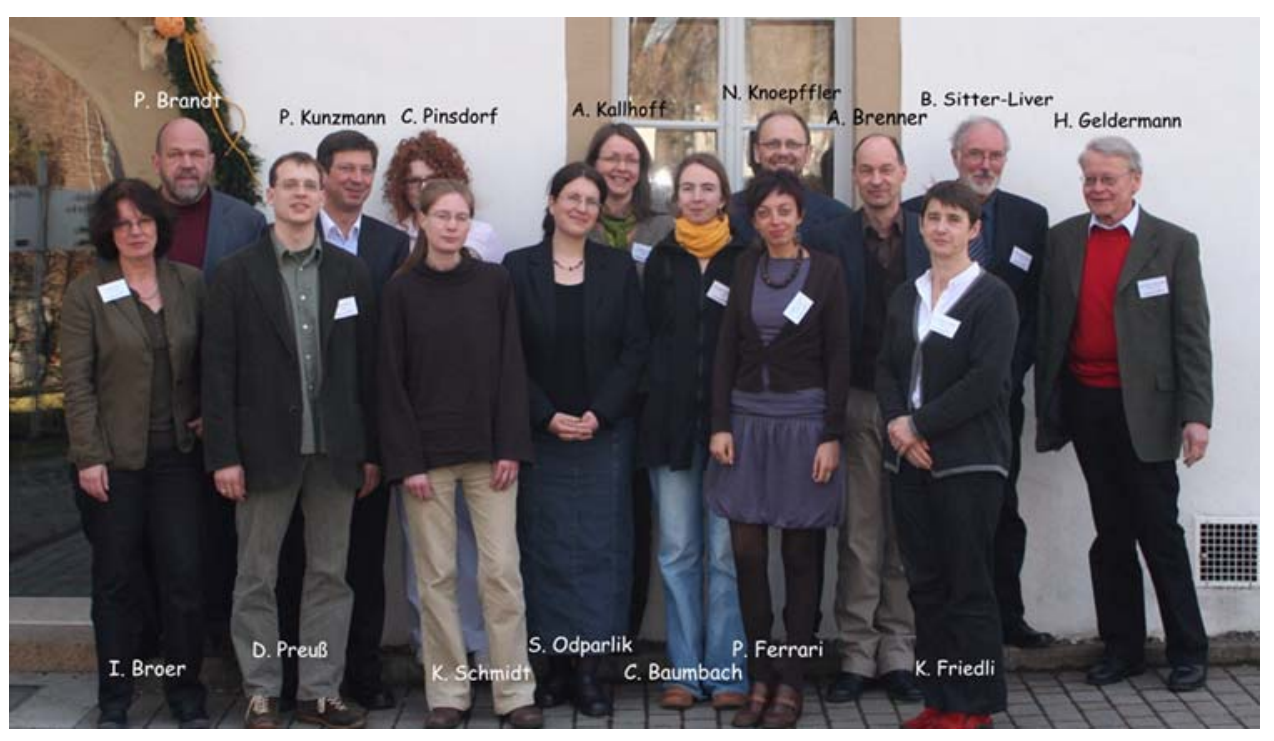

Der Streit um die Gentechnik scheint zu eskalieren, zumindest, wenn es um die so genannte Grüne Gentechnik, die Gentechnik an Pflanzen, geht. Umweltverbände rufen zu Massendemonstrationen auf und radikale Aktivisten zerstören Freisetzungsversuche, was sie selbst als "Feldbefreiung" feiern. Die Stars der Gentechnikgegner, wie Percy Schmeiser, füllen ganze Hallen bei ihren Vorträgen. Auf das Anbauverbot einer Mais-Sorte durch ein Bundesministerium

\footnotetext{
S. Odparlik ( $₫)$ P P. Kunzmann ( $\varangle)$

Friedrich-Schiller-Universität Jena, Bereich Ethik in den Wisseschaften, Zwätzengasse 3, 07743 Jena, Germany e-mail: sabine.odparlik@uni-jena.de

P. Kunzmann

e-mail: Peter.Kunzmann@uni-jena.de
}

reagiert ein anderes mit einem Gentechnikgipfel in Form eines Expertengesprächs.

Tiefe Gräben durchziehen die Diskurs-Landschaft, tiefer jedenfalls, als sie entlang von Fakten und Sachverhalten entstehen könnten. Offenkundig entladen sich im Streit um die Gentechnik sehr viel tiefer sitzende Urteile und Vorurteile, wofür die deutsche Sprache das schöne, mittlerweile international bekannte Wort "Weltanschauung" erfunden hat. Dies bietet Anlass genug, auf der Basis des aktuellen Sachstands Tiefenbohrungen in diejenige DenkSchichten vorzunehmen, wo dieser weltanschauliche Streit eigentlich ausgetragen und entschieden wird. Es geht sicherlich um mehr als Umweltschutz und Lebensmittelsicherheit, um mehr als Verbraucherschutz und Kennzeichnungspflicht. Es geht um einen 
ethisch fundierten moralischen Umgang mit nichtmenschlichen Lebewesen, der die verschiedenen Interessen und Perspektiven ausgewogen bewertet und entsprechend berücksichtigt.

Um diese interdisziplinären Untersuchungen sinnvoll vorzunehmen, existiert seit 2005 am Lehrstuhl für Angewandte Ethik an der FriedrichSchiller-Universität Jena eine Arbeitsgruppe zum Thema "Würde in der Gentechnologie", gefördert vom Bundesministerium für Bildung und Forschung. Diese Arbeitsgruppe hat auch die Veranstaltung “Würde versus Gentechnologie?” ausgerichtet, der die folgenden Beiträge im Wesentlichen entstammen und die als Klausur-Tagung vom 31. März bis 3. April 2009 im Schlosshotel Eyba bei Saalfeld/Thüringen stattfand. Philosophen und Theologen, Ethiker und Naturwissenschaftler behandelten die ethischen Implikationen der Gentechnik an Tieren und Pflanzen aus unterschiedlicher Perspektive, aber doch unter erkennbar einheitlichen Maßstäben.

Das gilt auch mit Blick auf eine zweite, eigentlich sonderbare Eigenschaft des aktuellen Streits um die Gentechnik: dass einerseits die Gentechnik am Tier relativ unbeachtet bleibt, während sie an der Pflanze massiven Protest hervorrufen kann. Gibt es nicht eine einheitliche, umfassendere ethische Rahmenhandlung, an der Menschen in ihrem Handeln Maß nehmen sollen, wenn sie in nicht-menschliches Leben eingreifen? Die Schweizer versuchten 1992 diesen ethischen Rahmen mit dem Verfassungsbegriff einer "Würde der Kreatur" abzustecken. Es bleiben zwar je besondere Fragen im Umgang mit Tieren und Pflanzen, es zeichnen sich aber auch einige grundlegende Prinzipien ab, die für Ethik und Moral mit Blick auf beide kennzeichnend sind. Gerade darin hat die Diskussion im letzten Jahrzehnt deutliche Konturen gewonnen. In den hier versammelten Beiträgen sind sie gut zu studieren: Während im Anfang noch ein unsicheres Changieren zwischen eher ökologisch und eher bioethisch fokussierten Ansätzen vorlag, besteht heute weit gehend Einigkeit, dass der Fluchtpunkt aller Überlegungen im individuellen Lebewesen zu sehen sei, seinem "Lebensdrive" (Knoepffler), seinem Streben nach Selbsterhalt und Selbstvollzug, seinem je eigenen
Gut. Zugleich hat sich mit der Kategorie der "Integrität” ein Instrument etabliert, den Respekt vor der Würde zugleich auf der einen Seite zu begründen, wie auf der anderen Seite zu präzisieren und zu konkretisieren, wo und wie genau diese Würde verletzt werden kann. Dabei sind sich die Autoren bewusst, dass diese Maßstäbe nicht nur an die gentechnische Veränderung von Lebewesen anzulegen sind, sondern manches Verfahren im so genannten konventionellen Bereich in neuem Licht erscheinen lässt und umgekehrt die Gentechnik gerade nicht in sich selbst einen neuen, besonders massiven Eingriff des Menschen in die Natur und die Naturdinge etablieren muss.

Die Diskussion um die "Würde der Kreatur" förderte also Prinzipien zu Tage, die es möglich machen, den sonst eher unterschwelligen Konflikt um des Menschen Zugriff auf fremdes Leben zu verbalisieren, $\mathrm{zu}$ strukturieren, $\mathrm{zu}$ diskutieren und $\mathrm{zu}$ entscheiden.

Dessen eingedenk waren vier Schritte nötig, die zugleich die Beiträge in diesem Journal gliedern: Auf einige grundlegende Überlegungen zum Thema der Würde der Kreatur (und ihrem Verhältnis zur Menschenwürde) folgt je ein eigener Bericht über den Sachstand in der Gentechnik bei Pflanzen bzw. bei Tieren. Von hier aus ist es möglich, die beiden Einsatzgebiete von Gentechnik in ethischer Perspektive zu sichten und zu bewerten. Die Schwerpunkte sind jeweils verschieden, doch wird der Leser übergreifende Argumente wiederfinden, Argumente die vor allem darauf abzielen, Tieren und Pflanzen in der Ethik jenen Status zuzuweisen, der für die moralische Beurteilung der Gentechnik sinnvoll und notwendig ist.

Unser Dank gilt dem Bundesministerium für Bildung und Forschung für die nachhaltige Unterstützung des Forschungsprojekts. Er geht an alle Mitwirkende des Unternehmens “Würde versus Gentechnologie?", ganz besonders aber an Herrn Professor Dr. Dr. Peter Brandt für seine Initiative und sein Engagement, das es ermöglicht hat, die Erträge in diesem Journal einem großen, sachkundigen Publikum zugänglich zu machen. 